\title{
AUTHOR INDEX \\ VOLUME 9 (1995)
}

Acharya, T. \& Mukherjee, A., High-speed parallel VLSI architectures for image decorrelation

9 (1995) 343-365

Ahmed, A., see Venkateswaran, N.

Al-Sadoun, H.B. \& Amin, A., A new structural technique for recognizing printed Arabic text

9 (1995) 231-262

Albanesi, M. \& Ferretti, M., Systolic merging and ranking of votes for the generalized hough transform

9 (1995) 101-125

9 (1995) 315-341

9 (1995) 101-125

Anigbogu, J.C. \& Belaïd, A., Hidden Markov models in text recognition

9 (1995) 925-958

9 (1995) 201-229

9 (1995) 421-447

9 (1995) 925-958

Belaïd, A., see Anigbogu, J.C.

Bhandarkar, S.M., A surface feature attributed hypergraph representation for 3-D recognition

Bhandarkar, S.M., Arabnia, H.R. \& Smith, J.W., A reconfigurable architecture for image processing and computer vision

9 (1995) 869-909

9 (1995) 201-229

Bhattacharya, A.K. \& Haider, S.S., A VLSI implementation of the inverse discrete cosine transform

9 (1995) 303-314

Bokka, V., Gurla, H., Olariu, S., Schwing, J.L. \& Stojmenović, I., Time-optimal digital geometry algorithms on meshes with multiple broadcasting

Borsack, J., see Taghva, K.

Bourbakis, N.G., Knowledge extraction and acquisition during real-time navigation in unknown environments

Bruckstein, A.M., Sapiro, G. \& Shaked, D., Evolutions of planar polygons

Bullard, B., see Taghva, K.

Catania, V. \& Ascia, G., A VLSI parallel architecture for fuzzy expert systems

Chaudhuri, B.B., see Sengupta, P.

Cheng, H.D. \& Cheng, X., Shape recognition using a fixed-size VLSI architecture

Cheng, X., see Cheng, H.D.

Ching, W.-S., A fast and effective occlusion detection algorithm

Chung, K.L., Fast pattern-matching algorithm on modular mesh-connected computers with multiple buses

Condit, A., see Taghva, K.

Dare, V.R., see Subramanian, K.G.

Dassow, J., Freund, R. \& Păun, G., Cooperating array grammar systems

Davis, L.S., Inoue, K., Nivat, M., Rosenfeld, A. \& Wang, P.S.P., Parallel image analysis: theory and applications - Part 1
9 (1995) 601-613
9 (1995) 911-923
$9(1995) 83-99$
9 (1995) 991-1014
9 (1995) 911-923
9 (1995) 421-447
o (1995) 1015-1028
9 (1995) 1-21
9 (1995) 1-21
9 (1995) 565-578
9 (1995) 411-419
9 (1995) 911-923
9 (1995) 763-775
9 (1995) 1029-1053
9 (1995) 599-600

1057 
Dawson-Howe, K.M. \& Vernon, D., 3-D object recognition through implicit model matching

de Bertrand de Beuvron, F. \& Trigano, P., Hierarchically coded lexicon with variants

Debled-Rennesson, I. \& Reveilles, J.-P., A linear algorithm for segmentation of digital curves

Dengel, A., Syntactic analysis and representation of spatial structures by puzzletrees

Desouza, J., see Venkateswaran, N.

Devanathan, R., see Venkateswaran, N.

Ferretti, M., see Albanesi, M.

Foresti, G.L., see Murino, V.

Freund, R., see Dassow, J.

Gerrard, J.M., see Gordon, H.

Ghosh, I. \& Majumdar, B., VLSI implementation of an efficient ASIC architecture for real-time rotation of digital images

Gordon, H., Pizzi, N.J., Gerrard, J.M. \& Somorjai, R., Assessment of bleeding predispositions in tonsillectomy/ adenoidectomy patients using non-metric clustering

Gurla, H., see Bokka, V.

Ha, J.-Y., Oh, S.-C. \& Kim, J.H., Recognition of unconstrained handwritten English words with character and ligature modeling

Haider, S.S., see Bhattacharya, A.K.

Herbordt, M.C. \& Weems, C.C., Enpassant: An environment for evaluating massively parallel array architectures for spatially mapped applications

Huang, Y.S., Liu, K. \& Suen, C.Y., The combination of multiple classifiers by a neural network approach

Inoue, K., see Davis L.S.

Inoue, K., see Ito, A.

Inoue, K., see Wang, Y.

Ito, A., Inoue, K., Takanami, I. \& Wang, Y., The effect of inkdots for two-dimensional automata

Iyengar, P.A., see Samal, A.

Kankanhalli, A., see Lee, C,-M.

Kim, J.H., see Ha, J.-Y.

Kong, T.Y., On topology preservation in 2-D and 3-D thinning

Kumaran, B., see Venkateswaran, N.

Lee, C.-M. \& Kankanhalli, A., Automatic extraction of characters in complex scene images

Liu, K., see Huang, Y.S.

Majumdar, B., see Ghosh, I.

Marion-Poty, V. \& Miguet, S., Data allocation strategies for parallel image processing algorithms

Marion-Poty, V., Two methodologies to implement 3D thinning algorithms on distributed memory machines

Mase, K., see Ueda, N.

Miguet, S., see Marion-Poty, V.

Mukherjee, A., see Acharya, T.

9 (1995) 959-990

9 (1995) 145-165

9 (1995) 635-662

9 (1995) 517-533

9 (1995) 263-301

9 (1995) 231-262

9 (1995) 315-341

9 (1995) 23-65

9 (1995) 1029-1053

9 (1995) 557-564

9 (1995) 449-462

9 (1995) 557-564

9 (1995) 601-613

9 (1995) 535-556

9 (1995) 303-314

9 (1995) 175-200

9 (1995) 579-597

9 (1995) 599-600

9 (1995) 777-796

9 (1995) 797-811

9 (1995) 777-796

9 (1995) 845-867

9 (1995) 67-82

9 (1995) 535-556

9 (1995) 813-844

9 (1995) 231-262

9 (1995) 67-82

9 (1995) 579-597

9 (1995) 449-462

9 (1995) 615-634

9 (1995) 699-717

9 (1995) 465-484

9 (1995) 615-634

9 (1995) 343-365 
Murino, V., Regazzoni, C.S., Foresti, G.L. \& Vernazza, G., A multilevel fusion approach to object identification in outdoor road scenes

Nakamura, A., Some notes on parallel coordinate grammars

Narayanan, S., see Venkateswaran, N.

Nivat, M., see Davis L.S.

Oh, S.-C., see Ha, J.-Y.

Olariu, S., see Bokka, V.

Pattabiraman, S., see Venkateswaran, N.

Pattabiraman, S., see Venkateswaran, N.

Paŭn, G., see Dassow, J.

Perroton, L., A new 26-connected objects surface tracking algorithm and its related PRAM version

Pizzi, N.J., see Gordon, H.

Radharamanan, see Venkateswaran, N.

Ranganathan, N., see Sastry, R.

Ranganathan, N., VLSI and parallel computing for pattern recognition and artificial intelligence

Regazzoni, C.S., see Murino, V.

Reveilles, J.-P., see Debled-Rennesson, I.

Roli, F., Serpico, S.B. \& Vernazza, G., Image recognition by integration of connectionist and symbolic approaches

Rosenfeld, A., see Davis L.S.

Samal, A. \& Iyengar, P.A., Human face detection using Silhouettes

Saoudi, A., see Senoussi, H.

Saoudi, A., see Subramanian, K.G.

Sapiro, G., see Bruckstein, A.M.

Sastry, R. \& Ranganathan, N., PMAC: A polygon matching chip

Schwing, J.L., see Bokka, V.

Sengupta, P. \& Chaudhuri, B.B., Projection of multi-worded lexical entities in an inflectional language

Senoussi, H. \& Saoudi, A., Quadtree algorithms for template matching on mesh connected computer

Serpico, S.B., see Roli, F.

Shaked, D., see Bruckstein, A.M.

Shankar, R., see Venkateswaran, N.

Sideras, M.A., see Ziavras, S.G.

Siromoney, R., see Subramanian, K.G.

Smith, J.W., see Bhandarkar, S.M.

Somorjai, R., see Gordon, H.

Srinivasan, R., see Venkateswaran, N.

Sriram, G., see Venkateswaran, N.

Stoica, I., A time-optimal multiple-query nearest-neighbour algorithm on meshes with multiple broadcasting

Stojmenović, I., see Bokka, V.

Subramanian, K.G., Siromoney, R., Dare, V.R. \& Saoudi, A., Basic puzzle languages

Suen, C.Y., see Huang, Y.S.

Suresh, G., see Venkateswaran, N.
9 (1995) 23-65

9 (1995) 753-761

9 (1995) 231-262

9 (1995) 599-600

9 (1995) 535-556

9 (1995) 601-613

9 (1995) 231-262

9 (1995) 263-301

9 (1995) 1029-1053

9 (1995) 719-734

9 (1995) 557-564

9 (1995) 231-262

9 (1995) 367-385

9 (1995) 173-174

9 (1995) 23-65

9 (1995) 635-662

9 (1995) 485-515

9 (1995) 599-600

9 (1995) 845-867

9 (1995) 387-410

9 (1995) 763-775

g (1995) 991-1014

9 (1995) 367-385

9 (1995) 601-613

9 (1995) 1015-1028

9 (1995) 387-410

9 (1995) 485-515

9 (1995) 991-1014

9 (1995) 263-301

9 (1995) 679-698

9 (1995) 763-775

9 (1995) 201-229

9 (1995) 557-564

9 (1995) 263-301

9 (1995) 263-301

9 (1995) 663-676

9 (1995) 601-613

9 (1995) 763-775

9 (1995) 579-597

9 (1995) 263-301 
Taghva, K., Borsack, J., Bullard, B. \& Condit, A., Post-editing through approximation and global correction

Takanami, I., see Ito, A.

Takanami, I., see Wang, Y.

Tanaka, E., A note on a tree-to-tree editing problem

Trigano, P., see de Bertrand de Beuvron, F.

Ueda, N. \& Mase, K., Tracking moving contours using energyminimizing elastic contour models

Venkateswaran, N., Pattabiraman, S., Desouza, J., Sriram, G., Srinivasan, R., Shankar, R. \& Suresh, G., A design methodology for very large array processors - Part 2:

Pacube VLSI arrays

Venkateswaran, N., Pattabiraman, S., Devanathan, R., Kumaran, B., Ahmed, A., Narayanan, S. \& Radharamanan, A design methodology for very large array processors - Part 1: GIPOP processor array

Vernazza, G., see Murino, V.

Vernazza, G., see Roli, F.

Vernon, D., see Dawson-Howe, K.M.

Wang, P.S.P., see Davis L.S.

Wang, P.S.P., see Zhang, Y.Y.

Wang, Y., Inoue, K. \& Takanami, I., Co-operating systems of three-way, two-dimensional finite automata

Wang, Y., see Ito, A.

Weems, C.C., see Herbordt, M.C.

Zamperoni, P., An adaptive rank filter for image enhancement depending on a measure of the local spatial order

Zhang, Y.Y. \& Wang, P.S.P., Analysis and design of parallel thinning algorithms - a generic approach

Ziavras, S.G. \& Sideras, M.A., Facilitating high-performance image analysis on reduced hypercube (RH) parallel computers

9 (1995) 911-923

9 (1995) 777-796

9 (1995) 797-811

9 (1995) 167-172

9 (1995) 145-165

9 (1995) 465-484

9 (1995) 263-301

9 (1995) 231-262

9 (1995) 23-65

9 (1995) 485-515

9 (1995) 959-990

9 (1995) 599-600

9 (1995) 735-752

9 (1995) 797-811

9 (1995) 777-796

9 (1995) $175-200$

9 (1995) 127-144

9 (1995) 735-752

9 (1995) 679-698 